\title{
Isolated resonances in conductance fluctuations in ballistic billiards
}

\author{
Arnd Bäcker ${ }^{\mathrm{a}}$, Achim Manze ${ }^{\mathrm{b}, 1}$, Bodo Huckestein ${ }^{\mathrm{b}}$, Roland Ketzmerick ${ }^{\mathrm{c}}$ \\ a Abteilung Theoretische Physik, Universität Ulm, Albert-Einstein-Allee 11, D-89081 Ulm, Germany \\ ${ }^{\mathrm{b}}$ Institut für Theoretische Physik III, Ruhr-Universität Bochum, D-44780 Bochum, Germany \\ ${ }^{\mathrm{c}}$ Max-Planck-Institut für Strömungsforschung and Institut für Nichtlineare Dynamik der Universität Göttingen, Bunsenstraße \\ 10, D-37073 Göttingen, Germany
}

\begin{abstract}
We study the isolated resonances occurring in conductance fluctuations of ballistic electron systems with a classically mixed phase space. In particular, we calculate the conductance and Wigner-Smith time as well as scattering states and eigenstates of the open and closed cosine billiard, respectively. We demonstrate that the observed isolated resonances and their scattering states can be associated with eigenstates of the closed system. They can all be categorized as hierarchical or regular, depending on where in a phase space representation the corresponding eigenstates are concentrated.
\end{abstract}

Key words: soft quantum chaos; semiclassical theories; scattering; billiard system

One of the properties in which classical chaos manifests itself quantum mechanically is the conductance. A well known example is the occurrence of the universal conductance fluctuations in billiards whose classical counterparts show completely chaotic dynamics [1]. However, generic systems are neither completely chaotic nor integrable but have a mixed phase space, where regions of regular motion coexist with those of chaotic motion [2]. A semiclassical analysis for the quantum mechanical analog of such systems showed that the graph of conductance $G$ vs control parameter should be a fractal [3] (fractal conductance fluctuations).

Surprisingly, for the cosine billiard, a system with a mixed phase space, a recent numerical study did not show these fractal conductance fluctuations but instead sharp isolated resonances with a width distribution covering several orders of magnitude [4] superimposed on universal conductance fluctuations of order unity. The resonances in the conductance are accompanied by equally sharp but much stronger resonances in the Wigner-Smith time. We will show that the ori-

\footnotetext{
${ }_{1}$ E-mail: amz@tp3.ruhr-uni-bochum.de
}

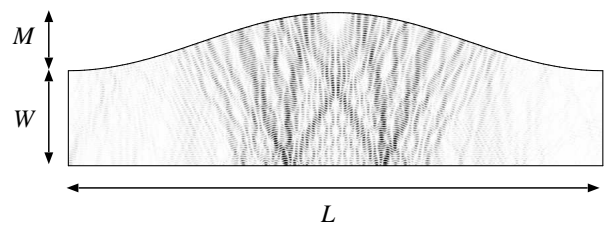

Fig. 1. The cosine billiard for the parameters $W / L=0.18$ and $M / L=0.11$ with a gray-scale plot of the density of the resonant scattering state leading to the Husimi representation shown in Fig. 3 b).

gins of these resonances are quantum mechanical states with phase space portraits that are concentrated on the regular and hierarchical regions of phase space [5], in aggreement with the conjecture of Ref. [6].

We study the cosine billiard as in Ref. [4] , either closed (hard wall boundaries at $x=0$ and $x=L$ ) or with semi-infinite leads attached to both sides. The boundary consists of the line $y=0$ and

$y(x)=W+\frac{M}{2}\left[1-\cos \left(\frac{2 \pi x}{L}\right)\right]$

for $0 \leq x \leq L$ (see Fig. 1). The classical phase space structure can be tuned by varying the ratios $W / L$ and 


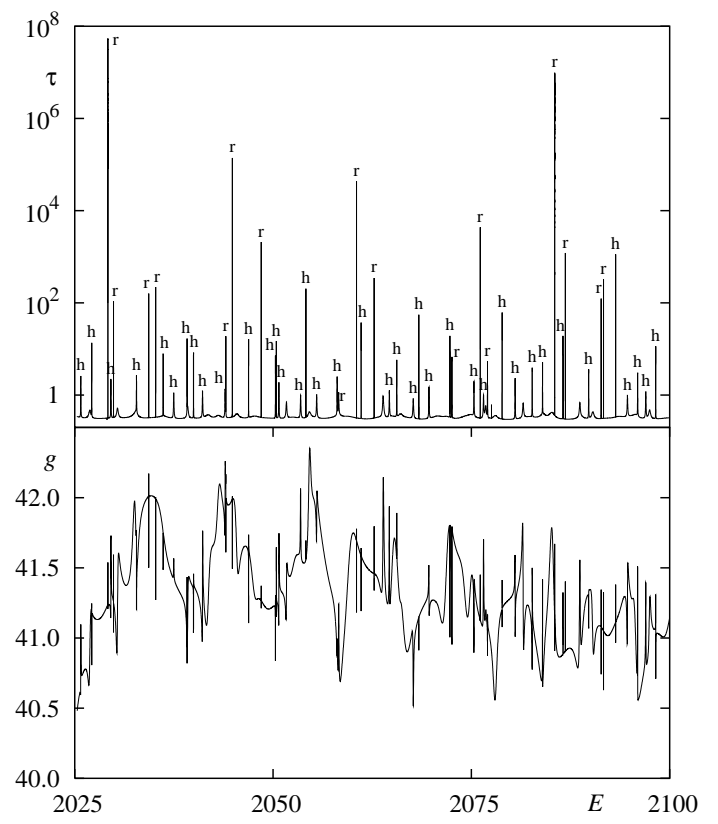

Fig. 2. Wigner-Smith delay time $\tau$ (upper part) and conductance $G$ (lower part) vs energy $E$. In the upper part, the labels of the resonances indicate whether they correspond to regular (r) or hierarchical (h) eigenstates of the closed system.

$M / L$. For $W / L=0.18$ and $M / L=0.11$ the system has a mixed phase space. We take $E_{0}=\hbar^{2} \pi^{2} /\left(2 m W^{2}\right)$ as the unit of energy.

Fig. 2 shows the isolated resonances occuring in both the conductance and the Wigner-Smith time delay $\tau=\frac{-i \hbar}{2 N} \operatorname{Tr}\left(S^{\dagger} d S / d E\right)(2 N$ is the dimension of the $S$-matrix). The calculations of $S$ and $\tau$ are outlined in Refs. [4,7].

In order to elucidate the origin of the resonances, we have calculated the associated scattering states of the open system as well as the eigenstates of the corresponding closed system. Fig. 3 shows a comparison of the Husimi representation of the eigenstates and scattering states with the classical phase space structure [7]. The energy of the scattering state coincides with the resonance energy 2041.109 and the eigenenergy of the eigenstate differs from this energy by less than $10 \%$ of the mean level spacing. It is apparent that this resonance is due to an eigenstate with a phase space representation concentrated on the hierarchical part of phase space. Repeating this analysis for the other resonances allows for them to be labeled as hierarchical or regular depending on the part of phase space that their Husimi representations are concentrated on. The resulting labels are shown in Fig. 2.

To conclude, we have analyzed phase space portraits of both eigenstates of a closed billiard and scattering states of the opened billiard. This allowed us to identify the origin of sharp, isolated resonances in the con- a)

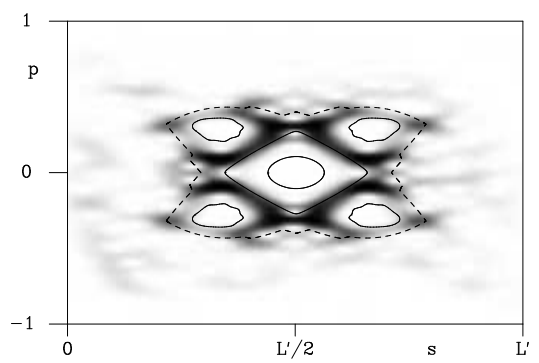

b)

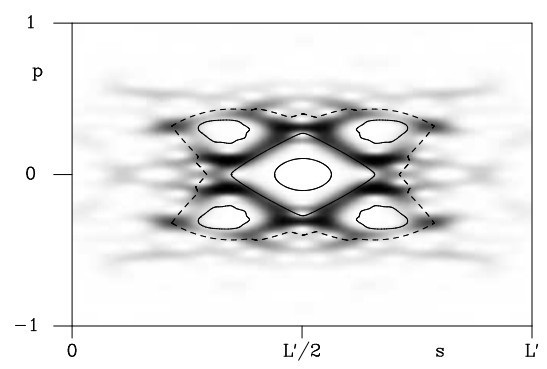

Fig. 3. (a) Husimi representation of a hierarchical eigenstate of the closed system. (b) A similar phase space representation of the corresponding scattering state (see also Fig. 1). Also shown are the most important Kolmogorov-Arnold-Moser tori (solid lines) and the main border to the chaotic region (dashed line).

ductance and Wigner-Smith time of the billiard and to classify the resonant states as either regular or hierarchical. In addition, we saw that the definition of hierarchical states of Ref. [5] can be transferred to scattering states as well.

Acknowledgements A.B. acknowledges support by the Deutsche Forschungsgemeinschaft under contract No. DFG-Ba 1973/1-1.

\section{References}

[1] R. A. Jalabert, in: Proceedings of the International School of Physics "Enrico Fermi", Course CXLIII New Directions in Quantum Chaos, G. Casati, I. Guarneri and U. Smilansky (eds.), IOS Press Amsterdam (2000).

[2] A. J. Lichtenberg, M. A. Liebermann, Regular and chaotic dynamics,, 2nd ed. (Springer-Verlag, New York, 1992).

[3] R. Ketzmerick, Phys. Rev. B 54 (1996) 10841.

[4] B. Huckestein, R. Ketzmerick, C. H. Lewenkopf, Phys. Rev. Lett. 84 (2000) 5504; ibid. 87 (2001) 119901(E); and references therein.

[5] R. Ketzmerick, L. Hufnagel, F. Steinbach, M. Weiss, Phys. Rev. Lett. 85 (2000) 1214.

[6] L. Hufnagel, M. Weiss, R. Ketzmerick, Europhys. Lett. 54 (2001) 703 . 
[7] A. Bäcker, A. Manze, B. Huckestein, R. Ketzmerick, Phys. Rev. E art. no. 016211 (2002). 